\title{
Probing the origin of thick disks using ultra-deep images
}

\author{
Cristina Martínez-Lombilla ${ }^{1,2}$ Ignacio Trujillo ${ }^{1,2}$ and \\ Johan H. Knapen ${ }^{1,2}$ \\ ${ }^{1}$ Instituto de Astrofísica de Canarias (IAC), La Laguna, 38205, Spain \\ ${ }^{2}$ Universidad de La Laguna (ULL), La Laguna, 38200, Spain
}

\begin{abstract}
The origin of thick disks is still a matter of debate. To explore such structures in unprecedented detail, we have developed a technique to reach a surface brightness limit of 28.5-29 mag/ $\operatorname{arcsec}^{2}$ with the combined $g, r, i$ bands images from the IAC Stripe82 Legacy Project (Fliri \& Trujillo, 2016). We present the characterisation of the thick disk in the edge-on galaxy UGC 01040. We carefully analyse the background subtraction and masking process. The effects of the PSF are considered through galaxy modelling. We present the study of radial and vertical surface brightness profiles, making a comparison between our data, the convolved and deconvolved models and its galactic components. We find that PSF effects are important, but can be accounted for. Our technique will allow us to model thick disks in external galaxies and elucidate their formation and evolutionary history.
\end{abstract}

Keywords. galaxies: evolution, galaxies: formation, galaxies: spiral, galaxies: structure.
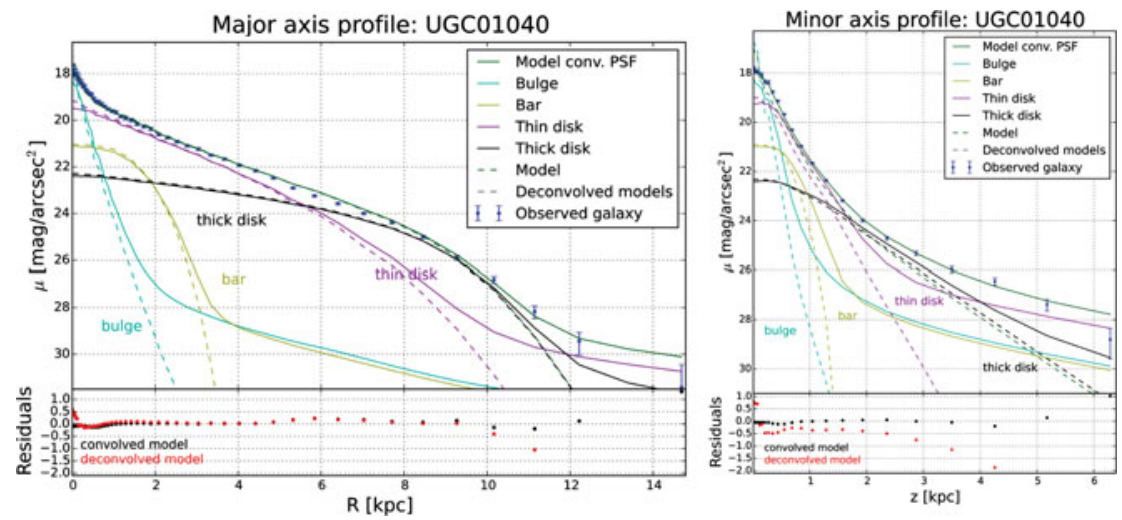

Figure 1. Left: Surface brightness profile along the major axis of the edge-on galaxy UGC 01040. We have plotted our data (blue dots), the model and its components. PSF-convolved components are shown with solid curves. A thick disk component is required to reach satisfactory fit results, which is dominant beyond $\sim 7 \mathrm{kpc}$. The PSF-affected thin disk is dominant in the very outskirts (beyond $12 \mathrm{kpc}$ ). Right: Surface brightness profile along the minor axis of UGC 01040. The deconvolved model is $\sim 1 \mathrm{mag} / \operatorname{arcsec}^{2}$ brighter than the data points in the centre. The effect of the PSF is relevant beyond $23 \mathrm{mag} / \operatorname{arcsec}^{2}$. The thick disk is the dominant component at $\mathrm{z}>2$ $\mathrm{kpc}$, but the PSF-affected thin disk is still dominant in the outskirts.

\section{Acknowledgments}

We acknowledge support from the Spanish Ministry of Economy and Competitiveness (MINECO) under grant number AYA2013-41243-P.

\section{Reference}

Fliri, J. \& Trujillo, I. 2016, MNRAS, 456, 1359 